\title{
Antecedent thermal injury worsens split-thickness skin graft quality: A clinically relevant porcine model of full-thickness burn, excision and grafting
}

\author{
Anders H. Carlsson, Ph.D. ${ }^{1,2}$, Lloyd F. Rose, Ph.D. ${ }^{1,2}$, John L. Fletcher, MD. ${ }^{1,2,3}$, Jesse C. Wu, \\ M.S. ${ }^{1,2}$, Kai P. Leung, Ph.D. ${ }^{2}$, Rodney K. Chan, M.D., F.A.C.S. ${ }^{1,2,3}$ \\ ${ }^{1}$ Q-SCARR ${ }^{\mathrm{TM}}$ (Quality Skin Collaborative for Advanced Reconstruction and Regeneration) \\ Research Program \\ ${ }^{2}$ Dental and Craniofacial Trauma Research and Tissue Regeneration, US Army Institute of \\ Surgical Research, 3650 Chambers Pass, JBSA Fort Sam Houston, TX 78234 \\ ${ }^{3}$ Clinical Division and Burn Center, US Army Institute of Surgical Research, 3650 Chambers \\ Pass, JBSA Fort Sam Houston, TX 78234
}

Corresponding Author:

Anders H. Carlsson, Ph.D.

E-mail address: anders.h.carlsson.ctr@mail.mil

Phone number: (210) 539-7422

Address: Dental and Craniofacial Trauma Research and Tissue Regeneration, US Army Institute of Surgical Research, 3650 Chambers Pass, JBSA Fort Sam Houston, TX 78234

This study has been conducted in compliance with the Animal Welfare Act, the implementing Animal Welfare Regulations, and the principles of the Guide for the Care and Use of Laboratory Animals.

The opinions or assertions contained herein are the private views of the authors are not to be construed as official or as reflecting the views of the Department of the Army or the Department of Defense. 


\section{Abstract}

Current standard of care for full-thickness burn injury is excision followed by autologous splitthickness skin graft placement. Skin grafts are also frequently used to cover surgical wounds not amenable to linear closure. While all grafts have potential to contract, clinical observation suggests that antecedent thermal injury worsens contraction and impairs functional and aesthetic outcomes. This study evaluates the impact of antecedent full-thickness burn on splitthickness skin graft scar outcomes and the potential mediating factors. Full-thickness contact burns $\left(100^{\circ} \mathrm{C}, 30\right.$ seconds) were created on the backs of anesthetized female Yorkshire Pigs. After seven days, burn eschar was tangentially excised and covered with $12 / 1000^{\text {th }}$ inch (300 $\mu \mathrm{m})$ split-thickness skin graft. For comparison, unburned wounds were created by sharp excision to fat before graft application. From 7 to 120 days post-grafting, planimetric measurements, digital imaging and biopsies for histology, immunohistochemistry and gene expression were obtained. At 120 days post-grafting, the Observer Scar Assessment Scale, colorimetry, contour analysis and optical graft height assessments were performed. Twenty-nine porcine wounds were analyzed. All measured metrics of clinical skin quality were significantly worse $(p<0.05)$ in burn injured wounds. Histological analysis supported objective clinical findings with marked scar-like collagen proliferation within the dermis, increased vascular density, and prolonged and increased cellular infiltration. Observed differences in contracture also correlated with earlier and more prominent myofibroblast differentiation as demonstrated by a-SMA staining. Antecedent thermal injury worsens split-thickness skin graft quality, likely by multiple mechanisms including burn-related inflammation, microscopically inadequate excision, and dysregulation of tissue remodeling. A valid, reliable, clinically relevant model of fullthickness burn, excision and skin replacement therapy has been demonstrated. Future research to enhance quality of skin replacement therapies should be directed toward modulation of inflammation and assessments for complete excision. 
Keywords : Burn, Scar, Contraction. 


\section{Introduction}

The current standard of care for treatment of full thickness burns is early excisional debridement and wound coverage, though this is not always possible [1]. Reasons include delayed presentation due to altered mental status [2, 3], prolonged transport time from remote points of injury [4], prioritization of life-saving treatments and intentional delay to await determination of uncertain injury depth [5]. While all split-thickness skin grafts (STSG) have the potential to contract, clinical observation suggests that antecedent local thermal injury and delayed eschar excision, exacerbate contraction and impair functional and aesthetic outcomes. Few studies, however, have thoroughly investigated the role of antecedent local full-thickness burn on STSG outcomes or the mechanisms that mediate these outcomes.

Several animal models of burn wound healing have been described [6, 7]. Similarities in skin biology between pig and human make porcine models ideal for translational research in both burn wound therapy and hypertrophic scar formation. However, most burn models allow the eschar to spontaneously slough - a practice largely abandoned in acute burn care [8-12]. Although spontaneous sloughing may be relevant for investigations of therapies to decrease burn progression or burn depth, the presence of burn eschar alters the wound healing microenvironment. Most studies also choose not to follow the wounds long enough to allow proper assessment of scar quality and contracture [13-18]. The animal model employed in this study differs notably from other porcine burn models because it more closely mimics current clinical strategy: excision of burn eschar followed by coverage with autologous STSG. Specifically, this study compares skin grafts placed on wound beds of similar depth after excision alone versus excision after antecedent burn. These groups were observed longitudinally for 120 days and excision for differences in macro-scale skin quality, histologic appearance and gene expression.

This study provides validation of a clinically relevant porcine model of tangential excision and grafting of burn wounds using both qualitative and quantitative measurements to assess scar 
quality. Understanding the contribution of antecedent full-thickness burn to STSG scar outcome will be valuable in developing novel strategies that mitigate the effect of burn eschar on STSG quality.

\section{Materials and Methods}

\section{Animals}

The use of 11 six-month old cross-bred female Yorkshire pigs (Midwest Research Swine, Gibbon, MN) was reviewed and approved by Institutional Animal Care and Use Committees (IACUC) at the United States Army Institute of Surgical Research (USAISR, JBSA Fort Sam Houston, TX). This study was conducted in compliance with the Animal Welfare Act, the implementing Animal Welfare Regulations, and the principles of the Guide for the Care and Use of Laboratory Animals. All animals received care in facilities accredited by Association for Assessment and Accreditation of Laboratory Animal Care International (AAALAC, Int.).

\section{Burn, Tangential Excision, and Grafting}

Five $6-\mathrm{cm}$ diameter wounds were tattooed bilaterally $(4 \mathrm{~cm}$ from each other and $2 \mathrm{~cm}$ from the spine) on the dorsal surface of the animal, totaling 10 wounds per animal. A total of 29 wounds, 15 burns and 14 excisions, were dedicated for this study and a controlled randomization was applied to assure equal representation from the five different dorsal wound locations. To control for animal growth, two additional 6-cm circles were tattooed but not excised. Total wound area, calculated using a porcine total body surface area formula [19], was less than $10 \%$. Fullthickness burns were created on anesthetized pigs with a brass cylinder ( $6 \mathrm{~cm}$ diameter) heated to $100^{\circ} \mathrm{C}$ and applied for 30 seconds. A thermocoupler maintained the temperature within $\pm 2^{\circ} \mathrm{C}$ throughout the duration of the burn. The surface of the brass block was calibrated to ensure even temperature distribution. Additionally when inflicting the burn, a measured weight was added to the device to ensure constant pressure (approximately $0.87 \mathrm{~N} / \mathrm{cm}^{2}$ ). After thermal 
insult, the wounds were dressed with Xeroform (Covidien, Mansfield, Massachusetts), cotton gauze, Ioban (3M Health Care, St. Paul, Minnesota), and a fabric vest (DeRoyal, Powell, Tennessee). The animals were then left to recover for 7 days, allowing burn eschar development. The burn eschar was then tangentially excised down to viable fat tissue and the wounds grafted using autologous skin from a hind limb donor site. Autologous Split-thickness skin grafts (STSG) were harvested using a Zimmer pneumatic dermatome (Zimmer Surgical, Inc., Dover, Ohio) adjusted to $12 / 1000^{\text {th }}$ of an inch $(\sim 300 \mu \mathrm{m})$. This skin graft thickness was chosen because it is commonly used in human skin grafting. The skin grafts were then cut to fit and sutured over the $6 \mathrm{~cm}$ defect. For comparison, on the same day as burn eschar excision and grafting, unburned normal skin was excised down to subcutaneous fat and autografted. Bolsters made from Xeroform and cotton gauze were used to hold the skin grafts in place and assure contact with the wound bed. The bolsters and backs of the animals were then covered with loban and a fabric vest.

\section{Observer Scar Assessment Scale (OSAS)}

The observer portion of the POSAS (Patient and Observer Scar Assessment Scale) was used since there was no patient participation during the assessment. Due to the lack of patient participation we renamed this modification to the POSAS the "Pig Observer Scar Assessment Scale". The scars were examined on day 120 post-grafting by three observers. Vascularity (degree of redness), relief (surface irregularity), and mobility (graft adherence to underlying surface) were assessed, compared to normal skin, and a score assigned for each parameter [20]. Briefly, selected OSAS variables were assigned a score from 1 to 10 , with a score of 1 reflecting close to normal skin and 10 representing the worst possible outcome. The remaining scar categories included in the original POSAS were assessed objectively.

\section{Objective Graft Analysis}


ImageJ software (National Institute of Health, Bethesda, MD) was used to calculate (1) contracture, defined as percent change in graft surface area normalized to growth control and (2) scar distortion (ratio of maximum length to maximum width of the graft on day 120). Direct measurement of graft elevation utilized a hand-held laser image capture device (SilhouetteMobile ${ }^{\text {TM }}$ Aranz Medical, New Zealand) to measure scar height. Erythema and Melanin Index (hemoglobin and melanin concentration, respectively) were assessed with the Cortex Technology DSMII Colorimeter (CyberDerm Inc., Broomall, PA).

\section{Histology}

On days 7, 14, 30,60, 90, and 120 post-grafting, $12 \mathrm{~mm}$ punch biopsies of the graft were collected and fixed in $10 \%$ neutral-buffered formalin, dehydrated and embedded in paraffin. Five-micrometer-thick sections were deparaffinized and stained with hematoxylin-eosin (H\&E), Masson's Trichrome (MTS) or picrosirius red (PSR). All sections were photographed (Eclipse 55i and DS-Fi1, Nikon, Melville, NY) under white light or plane-polarized light (PSR stain). On day 120, histological assessment of skin graft and measurement of scar thickness were performed. Results were presented as scar index, which is the ratio of scar thickness to normal dermal thickness. A higher number indicates increased scarring.

DNA fragmentation was detected using Click-iT TUNEL Alexa Fluor 488 Imaging Assay (Thermo Fisher Scientific, Waltham, MA) according to the manufacturer's instructions. Sections were mounted with ProLong Gold with DAPI (Thermo Fisher Scientific) and fluorescent images acquired using a Zeiss Axio Vert A1 (Zeiss, Oberkochen, Germany).

\section{Immunohistochemistry}

Tissues were cut into $5-\mu \mathrm{m}$ thick sections. After deparaffinization and hydration, sodium citrate (pH 6.0) antigen retrieval was performed and sections blocked with $10 \%$ normal goat serum for 
1 hour at room temperature. Sections were then treated with rabbit mAb a-smooth muscle actin (a-SMA) (Abcam, Cambridge, MA) and mouse mAb CD45 (Novus, Littleton, CO) primary antibodies, overnight at $4^{\circ} \mathrm{C}$. Endogenous peroxidase activity was blocked with $3 \%$ hydrogen peroxide. Primary antibody was detected with HRP-conjugated secondary antibody (goat antirabbit or goat anti-mouse) (Bio-Rad, Hercules, CA) followed by 3,3'-Diaminobenzidine (DAB) development (Vector Lab, Burlingame, CA). Slides were observed under Nikon Eclipse 55i light microscope and photographs taken with a Nikon DS-Fi1 camera.

\section{PCR}

Biopsies were taken on day 7 post-injury, flash frozen in liquid nitrogen and transferred to RNALater-ICE for storage at $-80^{\circ} \mathrm{C}$ prior to processing. Fifty micrograms of each biopsy sample was diced and transferred to $2 \mathrm{ml}$ of Qiazol Lysis reagent (Qiagen, Germany) and homogenized for $\sim 2$ minutes discontinuously at max speed in an Ultra-Turrax® T25 tissue homogenizer (IKALabortechnik, Germany). RNA was extracted with chloroform. Each sample was applied to a single RNeasy column (Qiagen, Germany) and processed according to manufacturer's protocol. RNA concentration was analyzed on a NanoDrop 2000C Spectrophotometer (Thermo Fisher Scientific) and $1 \mu \mathrm{g}$ of RNA was converted to cDNA using iScript ${ }^{\mathrm{TM}}$ CDNA Synthesis Kit (BioRad). One microliter of cDNA was analyzed in triplicate by RT-PCR using $\mathrm{QQ}^{\mathrm{TM}}$ SYBR® Green Supermix on a StepOnePlus RT-PCR System (Applied Biosystems/Life Technologies, Carlsbad, CA). Data was reported as fold change over unwounded skin and analyzed by the Pfaffl Method [21].

\section{Statistical Analysis}

Statistical differences for OSAS scoring, colorimetry, and graft dimensions were determined by two-way ANOVA with Tukey-Kramer adjustment, or nonparametric comparison for all pairs, by the Steel-Dwass method when appropriate, using JMP statistics software (SAS, Cary, NC). Gene expression data were analyzed with unpaired t-tests after Kolmogorov-Smirnov or 
D’Agostino-Pearson tests, where appropriate. Results are reported as mean \pm standard error of the mean (SEM). Significance was defined by $p<0.05$.

\section{Results}

\section{Collagen stain confirmed full-thickness burn injury}

Burn depth was assessed by three methods: (1) Hematoxylin-Eosin (H\&E) stain (2) Masson's Trichrome stain (MTS) of heat-denatured collagen and (3) TUNEL staining of DNA fragmentation. Tissue biopsies from 7 days post-burn revealed disruption of dermal cellular architecture (H\&E) and dark purple MTS staining through the entire dermis (Figure 1). By contrast, normal unburned skin showed blue, loose and wavy mature collagen. Heat damaged collagen appears red or purple instead of blue which normal collagen displays [22]. TUNEL staining confirmed fragmented DNA throughout the entire dermis. Based on these results on post-burn day 7 , our $100^{\circ} \mathrm{C} 30$-second contact burn resulted in full thickness injury.

\section{Day 120 burn wound graft skin quality is worse than excisional wound graft skin quality}

On day 120 post-grafting, quantitative assessments of graft healing were performed: contraction, distortion, graft elevation, scar index, colorimetric measurements of erythema and melanin, as well as qualitative observer scores for vascularity, relief, and mobility (Table 1). Compared to grafted excisional wounds, all available metrics of skin quality (contracture, scar distortion, scar elevation, discoloration, hypervascularity and tissue immobility) in grafted burn wounds were significantly worse (Table 1; Figure 2). In addition, consistent with objective clinical assessments of increased erythema, optical analysis suggested elevated hemoglobin and melanin concentrations. The burn group further demonstrated more surface irregularities (higher relief score) consistent with the graft elevation and scar index measurements. Finally, higher mobility scores in the burn group indicate a more adherent and immobile skin graft. When taken together comparing grafts from burn and excision wounds, the higher total OSAS 
scores of the burn-group skin grafts confirm decreased skin quality. These findings mirror the poor skin quality outcomes observed in humans.

\section{Distinct histopathologic features in grafted burns}

At day 120 post-grafting, H\&E staining of the burn group revealed findings consistent with increased cellular fibrosis. While cellular infiltration remained low in the excision group, the burn group demonstrated markedly increased cellular infiltration (Figure 3). In MTS, blue staining intensity increases with increasing collagen density. In picrosirius (PSR) staining of collagen, change in color is associated with changes in fiber density and organization [23]. As collagen fibers mature and arrange in thicker bundles, PSR color progressively changes from green to orange and to yellow. Normal tissue collagen appears mature and organized in typical mesh pattern. PSR staining of the grafted burn group revealed large amounts of thin and densely packed green fibers (pale blue in MTS) in the deeper dermis demonstrating immature collagen. The upper dermis showed very thick bundles of yellow to orange irregularly-arranged collagen. This type of collagen structure is seen in hypertrophic scars. By contrast, in the grafted excision group the deeper dermis has primarily yellow to green loose fibers without excessive collagen deposition. The upper dermis has less of the dense irregular collagen fibers.

The burn group also presents a distinct immunohistochemical profile. As shown with a-SMA staining, while the grafted excision group demonstrates very minimal myofibroblast infiltration, myofibroblast levels in the grafted burn wounds surged early. In addition, up through day 90 post-grafting, the staining intensity increased. Also by day 90 , as shown by dense pericyte staining around blood vessels in the superficial dermis, the burn group is hypervascularized. CD45 positive staining was used to identify leukocytes and the burn group shows a marked increase in leukocyte infiltration at day 60 that remained increased out to day 120 . 


\section{Gene Expression Profile}

When compared to grafted excisional wounds, grafted burn wounds expressed significantly higher levels of pro-inflammatory cytokines (IL-8, $\mathrm{p}<0.05$, and IL-1 $\beta, p<0.01$ ) at 7 days after wound creation. Also, in grafted burn wounds the extracellular matrix (ECM) remodeling protein Matrix Metalloproteinase 1 (MMP1, $\mathrm{p}<0.01$ ) was significantly suppressed (Figure 4). 


\section{Discussion}

Full thickness burns are known to result in poor functional and aesthetic outcomes [24, 25]. However, the contribution of antecedent local full thickness burn to the quality of split thickness skin grafts is not well described. Based on clinical observation, the presence of antecedent burn injury appears to worsen the outcome of split thickness skin graft. Function-limiting contractures and hypertrophic scars necessitating years of reconstructive operations [26] are common and serve to highlight the primary gap in burn reconstruction: the absence of an off-the-shelf skin replacement that reconstitutes micro- and macro-skin function, restores the aesthetic qualities of normal skin with limited donor site morbidity [27-29].

While the depth of cellular death can be readily determined with techniques such as TUNEL staining, the extent of cellular injury following burn is much less clear. Burn injury magnifies this problem by producing a gradient of injury that mirrors the propagation of heat through tissues. Despite the frequent use of discrete terms such as "zone of coagulation", "zone of stasis", and "zone of hyperemia" there is no objective method for determining the transition between these zones. Further, the precise cellular, transcriptional and translational changes found in each "zone" remain enigmatic. While burn-related necrosis may not reach the hypodermis, it is reasonable to assume sub-lethal thermal injury may impair hypodermal tissues. Considering recent evidence that STSGs placed on fat undergo less contraction than those placed on fascia [30] this sub-lethal thermal injury may attenuate the role of fat in ameliorating contracture in this model.

In this study, we have demonstrated that antecedent burn injury adversely influences all tested metrics of skin graft quality and these findings are correlated with local elevations of proinflammatory cytokines and a persistent CD45 positive cell infiltrate. Compared to grafts on 
excisional wounds, grafts placed on tangentially excised burn wounds contract more than twice as much. While this difference is stark it fails to adequately describe the disfigurement seen clinically. In fact, by using distortion as a novel metric of disfiguring contracture we were able to further quantify the difficult to measure phenomenon of axial contracture. Together, differences in contracture and distortion highlight the tremendous influence antecedent burn have on skin graft contracture.

Graft contraction occurs when fibroblasts, in response to inflammatory signals such as TGF- $\beta$ and PDGF, differentiate into myofibroblasts [31, 32]. Actin-mediated contracture of myofibroblasts narrows wound edges and leads to irregularities in wound contour. This phenomenon is evident in our subjective evaluation of graft surface topography (i.e., relief). Grafts from the burn group were consistently given a higher relief score, reflecting the ruffled appearance. Corroborating this finding, objective measurements of graft elevation were two-fold higher when compared to excision alone. This confirms that following burn and excision a thicker scar develops under the graft. Some may suggest that the differences seen in scar thickness are due to normal anatomic variations in dermal thickness and that direct comparisons of scar thicknesses may give misleading results. Therefore we established a scar index; defined as the ratio of scar thickness to normal dermal thickness at the same location. Even after indexing, the observed differences remained statistically significant, indicating thicker scars in the burn group. As noted in the literature, burn scar contraction and scar thickness are correlated with high a-SMA expression [33]. Likewise in skin tissue from the burn group, we observed more $\alpha$-SMA positive staining. Thus the graft contraction we see is likely mediated by a large myofibroblast population.

Not surprisingly these thicker scars demonstrate reduced mobility and increased stiffness, reflecting differences in connective tissue deposition. Relative to the underlying surface, the 
burn group was significantly stiffer and more adherent (higher mobility score). The higher mobility score of the burn-group scars suggests prolific collagen deposition; consistent with our histological observations (H\&E and MTS). The presence of green PSR staining in the deep scar tissue of the burn group indicates that immature collagen is increased. In the upper part of the scar dermis, we observe thick mature collagen fibers in a striated orientation. By contrast, the excision group was composed of mature mesh-pattern collagen [23, 34]. Our observations suggest that the burn wounds developed a scar composed of thick layers of both mature striated collagen and unorganized immature collagen. This may result from excessive collagen production, decreased matrix degradation, or both.

Robust revascularization, critical for early skin graft survival, may ultimately lower skin quality and worsen scars. This is supported by the frequently observed late hypervascularity [35-37] seen in hypertrophic scars. In this study we observed a higher vascular score in burn group wounds, supporting this hypothesis. In addition, reflecting increased microvasculature, day 120 scars had both elevated erythema measurements and increased pericyte staining. During early time points in normal wound healing, elevated metabolic requirements require excess vasculature. However, once the proliferation phase is complete and the metabolic requirements are met, the excess blood vessels should be degraded [38]. In this study, microvascular networks in burn wounds were abundant, even at 120 days. This failure of the microvasculature to regress suggests a disruption to normal wound healing processes. It stands to reason that changes in expression of angiogenic factors are responsible for improper persistence of the microvasculature. As previously reported, stimulation of angiogenesis by VEGF can improve healing rates [39-45]. However, the effects of VEGF on scarring are not well understood. There is evidence that VEGF-induced angiogenesis could indirectly promote scar tissue deposition [46]. Alternatively, new endothelial cells could produce large quantities of profibrotic factors like 
TGF- $\beta$ and connective tissue growth factor (CTGF); these factors could in turn stimulate dermal fibroblasts to synthesize excessive amounts of collagen.

Similarly, inadequate excision may contribute to post-burn contracture. In the absence of a reliable tool for assessing wound bed viability surgeons are obligated to excise burned tissue to a "bleeding" and presumably healthy wound bed. Though it is the current clinical standard, subjective excisions may leave burn-injured and necrotic tissues in the wound bed. Sub-lethally injured and necrotic tissues contribute to an inflammatory milieu, pro-fibrotic signaling, and graft failure - all conditions which promote contracture.

While the pathophysiology of poor scar outcomes following burn is multifactorial and incompletely understood, acute inflammatory infiltration has been strongly linked to fibrogenesis and hypertrophic scarring [47]. Acute inflammatory activation may be the result of a systemic inflammatory response syndrome (SIRS), the injury itself, delayed eschar excision, or incomplete excision. Commonly seen in large surface area burns and among combat casualties [48] robust SIRS is less likely in small surface area burns. In this model, with an overall wound burden of $<10 \%$ TBSA, observed changes in inflammatory mediators and alterations in gene expression are attributable to local inflammation. As others have reported, seven days after burn, we observed a significant increase in pro-inflammatory cytokines IL-8 and IL-1 $\beta$ gene expression in wound tissue biopsies [49-51]. While not a new finding, this pro-inflammatory milieu promotes a pro-fibrotic cellular profile by promoting fibroblast migration and differentiation. These findings may help explain why burn is a strong independent predictor of scarring $[52,53]$.

Less well understood is the role of late inflammatory cellular dysregulation in burn-related scarring. Interestingly, at day 60 in the burn group, we saw increased CD45+ (T-lymphocyte) 
inflammatory cellular infiltrates. This persistent T-lymphocytic infiltrate, previously implicated in scarring [54], may directly or indirectly contribute to the pro-fibrotic response following burn injury. Additionally, observed reductions of MMP1 gene expression suggest a decreased collagen degradation capacity, further impairing scar remodeling. Corroborating this concept, MMP1 expression has also been observed in human skin hypertrophic scar tissue $[55,56]$. Together, promotion of pro-fibrotic cellular wound ecology and persistent dysregulation of collagen remodeling could promote excessive scar formation.

\section{Conclusion}

Grafts placed on tangentially excised burn wounds result in worse skin graft quality than those placed on non-burned excisions of the same depth. Acute and chronic inflammation, failure of microvascular regression, sub-lethal cellular injury and disordered collagen production are likely contributors to the poor functional and aesthetic outcomes seen clinically. This model provides a valuable tool for objective in vivo evaluation of current and future skin replacement technologies. Further research into molecular mechanisms that impair skin graft quality, strategies to reduce eschar toxicity, and instruments that improve wound bed preparation are needed.

\section{Acknowledgement}

The authors would like to express their sincere gratitude to James $\mathrm{K}$. Aden, $\mathrm{PhD}$ for his assistance with statistical analysis of the data and Lingamanaidu V. Ravichandran, PhD for his editorial assistance and preparation of the manuscript.

This study was funded by the Department of Defense (DoD) and was supported in part by an appointment to the Postgraduate Research Participation Program at the U.S Army Medical Research and Materiel Command by the Oak Ridge Institute for Science and Education through an interagency agreement between the U.S Department of Energy and USAMRMC. 


\section{References}

[1] Nguyen TT, Gilpin DA, Meyer NA, Herndon DN. Current treatment of severely burned patients. Ann Surg. 1996;223:14-25.

[2] Thombs BD, Bresnick MG, Magyar-Russell G. Who attempts suicide by burning? An analysis of age patterns of mortality by self-inflicted burning in the United States. General hospital psychiatry. 2007;29:244-50.

[3] Cameron DR, Pegg SP, Muller M. Self-inflicted burns. Burns. 1997;23:519-21.

[4] Wolf SE, Kauvar DS, Wade CE, Cancio LC, Renz EP, Horvath EE, et al. Comparison between civilian burns and combat burns from Operation Iraqi Freedom and Operation Enduring Freedom. Ann Surg. 2006;243:786-92; discussion 92-5.

[5] Prasanna M, Mishra P, Thomas C. Delayed primary closure of the burn wounds. Burns. 2004;30:169-75.

[6] Domergue S, Jorgensen C, Noel D. Advances in Research in Animal Models of BurnRelated Hypertrophic Scarring. J Burn Care Res. 2014.

[7] Sullivan TP, Eaglstein WH, Davis SC, Mertz P. The pig as a model for human wound healing. Wound repair and regeneration : official publication of the Wound Healing Society [and] the European Tissue Repair Society. 2001;9:66-76.

[8] Cuttle L, Kempf M, Phillips GE, Mill J, Hayes MT, Fraser JF, et al. A porcine deep dermal partial thickness burn model with hypertrophic scarring. Burns. 2006;32:806-20.

[9] Singer AJ, McClain SA. A porcine burn model. Methods in molecular medicine. 2003;78:10719.

[10] Singer AJ, Berruti L, Thode HC, Jr., McClain SA. Standardized burn model using a multiparametric histologic analysis of burn depth. Academic emergency medicine : official journal of the Society for Academic Emergency Medicine. 2000;7:1-6.

[11] Singer AJ, Hirth D, McClain SA, Crawford L, Lin F, Clark RA. Validation of a vertical progression porcine burn model. J Burn Care Res. 2011;32:638-46.

[12] Wang X, Kimble RM. A review on porcine burn and scar models and their relevance to humans. Wound Practice and Research. 2010;18:41-9.

[13] Branski LK, Mittermayr R, Herndon DN, Norbury WB, Masters OE, Hofmann M, et al. A porcine model of full-thickness burn, excision and skin autografting. Burns. 2008;34:1119-27.

[14] Graham JS, Schomacker KT, Glatter RD, Briscoe CM, Braue EH, Jr., Squibb KS. Efficacy of laser debridement with autologous split-thickness skin grafting in promoting improved healing of deep cutaneous sulfur mustard burns. Burns. 2002;28:719-30. 
[15] Albritton A, Leonard DA, Leto Barone A, Keegan J, Mallard C, Sachs DH, et al. Lack of cross-sensitization between alpha-1,3-galactosyltransferase knockout porcine and allogeneic skin grafts permits serial grafting. Transplantation. 2014;97:1209-15.

[16] Chan QE, Harvey JG, Graf NS, Godfrey C, Holland AJ. The correlation between time to skin grafting and hypertrophic scarring following an acute contact burn in a porcine model. J Burn Care Res. 2012;33:e43-8.

[17] Greenwood JE, Dearman BL. Comparison of a sealed, polymer foam biodegradable temporizing matrix against Integra $(R)$ dermal regeneration template in a porcine wound model. $J$ Burn Care Res. 2012;33:163-73.

[18] Schittek A, Demetriou AA, Seifter E, Stein JM, Levenson SM. Microcrystalline collagen hemostat (MCCH) and wound healing. Ann Surg. 1976;184:697-704.

[19] Swindle MM, Makin A, Herron AJ, Clubb FJ, Jr., Frazier KS. Swine as models in biomedical research and toxicology testing. Vet Pathol. 2012;49:344-56.

[20] Draaijers LJ, Tempelman FR, Botman YA, Tuinebreijer WE, Middelkoop E, Kreis RW, et al. The patient and observer scar assessment scale: a reliable and feasible tool for scar evaluation. Plast Reconstr Surg. 2004;113:1960-5; discussion 6-7.

[21] Pfaffl MW. A new mathematical model for relative quantification in real-time RT-PCR. Nucleic Acids Res. 2001;29:e45.

[22] Chvapil M, Speer DP, Owen JA, Chvapil TA. Identification of the depth of burn injury by collagen stainability. Plast Reconstr Surg. 1984;73:438-41.

[23] Rich L, Whittaker P. Collagen and Picrosirius Red Staining: A Polarized Light Assessment of Fibrillar Hue and Spatial Distribution. Brazilian Journal of Morphological Sciences. 2005;22:97-104.

[24] Benson A, Dickson WA, Boyce DE. Burns. Bmj. 2006;332:649-52.

[25] Donelan MB. Grabb and Smith's Plastic Surgery. In: Thorne CH, editor. 6th ed: Lippincott Williams \& Wilkins; 2006. p. 960.

[26] Chan RK, Aden J, Wu J, Hale RG, Renz EM, Wolf SE. Operative utilization following severe combat-related burns. Journal of burn care \& research : official publication of the American Burn Association. 2015;36:287-96.

[27] Proksch E, Brandner JM, Jensen JM. The skin: an indispensable barrier. Experimental dermatology. 2008;17:1063-72.

[28] Madison KC. Barrier function of the skin: "la raison d'etre" of the epidermis. J Invest Dermatol. 2003;121:231-41.

[29] Burkitt HG, Young B, Heath JW. Wheater's Functional Histology. 3rd ed. New York, NY: Churchill Livingstone; 1993. 
[30] Rose LF, Wu JC, Carlsson AH, Tucker DI, Leung KP, Chan RK. Recipient wound bed characteristics affect scarring and skin graft contraction. Wound Repair Regen. 2015.

[31] Watts GT, Grillo HC, Gross J. Studies in Wound Healing: II. The Role of Granulation Tissue in Contraction. Ann Surg. 1958;148:153-60.

[32] Gross J, Farinelli W, Sadow P, Anderson R, Bruns R. On the mechanism of skin wound "contraction": a granulation tissue "knockout" with a normal phenotype. Proc Natl Acad Sci U S A. 1995;92:5982-6.

[33] Wang XQ, Kravchuk O, Winterford C, Kimble RM. The correlation of in vivo burn scar contraction with the level of alpha-smooth muscle actin expression. Burns. 2011;37:1367-77.

[34] Szendroi M, Vajta G, Kovacs L, Schaff Z, Lapis K. Polarization colours of collagen fibres: a sign of collagen production activity in fibrotic processes. Acta Morphol Hung. 1984;32:47-55.

[35] van der Veer WM, Niessen FB, Ferreira JA, Zwiers PJ, de Jong EH, Middelkoop E, et al. Time course of the angiogenic response during normotrophic and hypertrophic scar formation in humans. Wound Repair Regen. 2011;19:292-301.

[36] Wang J, Chen H, Shankowsky HA, Scott PG, Tredget EE. Improved scar in postburn patients following interferon-alpha2b treatment is associated with decreased angiogenesis mediated by vascular endothelial cell growth factor. Journal of interferon \& cytokine research : the official journal of the International Society for Interferon and Cytokine Research. 2008;28:423-34.

[37] Shen R, Li TZ, Qi SH, Liang HZ, Xu YB, Xie JL, et al. [A research of endothelial celltargeted therapy for cure of hypertrophic scar]. Zhonghua zheng xing wai ke za zhi = Zhonghua zhengxing waike zazhi $=$ Chinese journal of plastic surgery. 2003;19:254-7.

[38] Davis GE, Saunders WB. Molecular balance of capillary tube formation versus regression in wound repair: role of matrix metalloproteinases and their inhibitors. The journal of investigative dermatology Symposium proceedings / the Society for Investigative Dermatology, Inc [and] European Society for Dermatological Research. 2006;11:44-56.

[39] Deodato B, Arsic N, Zentilin L, Galeano M, Santoro D, Torre V, et al. Recombinant AAV vector encoding human VEGF165 enhances wound healing. Gene Ther. 2002;9:777-85.

[40] Galiano RD, Tepper OM, Pelo CR, Bhatt KA, Callaghan M, Bastidas N, et al. Topical vascular endothelial growth factor accelerates diabetic wound healing through increased angiogenesis and by mobilizing and recruiting bone marrow-derived cells. Am J Pathol. 2004;164:1935-47.

[41] Liu PY, Tong W, Liu K, Han SH, Wang XT, Badiavas E, et al. Liposome-mediated transfer of vascular endothelial growth factor cDNA augments survival of random-pattern skin flaps in the rat. Wound Repair Regen. 2004;12:80-5.

[42] Giunta RE, Holzbach T, Taskov C, Holm PS, Konerding MA, Schams D, et al. AdVEGF165 gene transfer increases survival in overdimensioned skin flaps. The journal of gene medicine. 2005;7:297-306. 
[43] Taub PJ, Marmur JD, Zhang WX, Senderoff D, Nhat PD, Phelps R, et al. Locally administered vascular endothelial growth factor CDNA increases survival of ischemic experimental skin flaps. Plast Reconstr Surg. 1998;102:2033-9.

[44] Galeano M, Deodato B, Altavilla D, Cucinotta D, Arsic N, Marini H, et al. Adeno-associated viral vector-mediated human vascular endothelial growth factor gene transfer stimulates angiogenesis and wound healing in the genetically diabetic mouse. Diabetologia. 2003;46:54655.

[45] Romanowski EG, Yates KA, Gordon YJ. Topical corticosteroids of limited potency promote adenovirus replication in the Ad5/NZW rabbit ocular model. Cornea. 2002;21:289-91.

[46] Wilgus TA, Ferreira AM, Oberyszyn TM, Bergdall VK, Dipietro LA. Regulation of scar formation by vascular endothelial growth factor. Lab Invest. 2008;88:579-90.

[47] Wynn TA. Fibrotic disease and the $T(H) 1 / T(H) 2$ paradigm. Nat Rev Immunol. 2004;4:58394.

[48] Deitch EA, Wheelahan TM, Rose MP, Clothier J, Cotter J. Hypertrophic burn scars: analysis of variables. J Trauma. 1983;23:895-8.

[49] Ou S, Liu GD, Tan Y, Zhou LS, Bai SR, Xue G, et al. A time course study about gene expression of post-thermal injury with DNA microarray. Int J Dermatol. 2015;54:757-64.

[50] Rani M, Zhang Q, Schwacha MG. Gamma delta T cells regulate wound myeloid cell activity after burn. Shock. 2014;42:133-41.

[51] Shen L, Cui Z, Lin Y, Wang S, Zheng D, Tan Q. Anti-inflammative effect of glycyrrhizin on rat thermal injury via inhibition of high-mobility group box 1 protein. Burns. 2015;41:372-8.

[52] Jeschke MG, Boehning DF, Finnerty CC, Herndon DN. Effect of insulin on the inflammatory and acute phase response after burn injury. Crit Care Med. 2007;35:S519-23.

[53] Arturson G. Pathophysiology of the burn wound. Annales chirurgiae et gynaecologiae. 1980;69:178-90.

[54] Wong VW, Paterno J, Sorkin M, Glotzbach JP, Levi K, Januszyk M, et al. Mechanical force prolongs acute inflammation via T-cell-dependent pathways during scar formation. FASEB J. $2011 ; 25: 4498-510$.

[55] Rohani MG, Parks WC. Matrix remodeling by MMPs during wound repair. Matrix biology : journal of the International Society for Matrix Biology. 2015;44-46C:113-21.

[56] Eto H, Suga H, Aoi N, Kato H, Doi K, Kuno S, et al. Therapeutic potential of fibroblast growth factor-2 for hypertrophic scars: upregulation of MMP-1 and HGF expression. Lab Invest. 2012;92:214-23. 
Figure Legends

Figure 1. Full-thickness injury confirmed by Hematoxylin and Eosin (H\&E), Masson's Trichrome (MTS) and TUNEL staining of pig skin 7 days after $100^{\circ} \mathrm{C}, 30$ second burn. Loss of dermal and cellular architecture is seen in H\&E while heat-denatured and coagulated collagen is shown by dark purple MTS. Normal collagen (blue) is seen at the burn wound border (red arrowhead) and in the uninjured control. Full-thickness cellular injury is confirmed by evidence of DNA fragmentation (green) in TUNEL staining (Scale bars: H\&E and MTS 1000 $\mu \mathrm{m}$; TUNEL $200 \mu m)$.

Figure 2. Appearance of 0.012 inch $(300 \mu \mathrm{m})$ autologous split-thickness skin grafts from burn and excision groups on day 14, 60, and 120 post-grafting. Graft from the burn group contracted significantly more than excision group.

Figure 3. Histological comparison between burn and excision groups. H\&E: hematoxylin \& eosin stain on day 120 post-grafting (magnification: 20x). There is a marked increase in collagen content in the burn compared to the excision group. There is also a significant amount of fibrocytes in the burn group. MTS: Masson's Trichrome stain for collagen on day 120 (20x). In the burn group, there is significant deposition of immature collage (pale blue). In the excision group, the collagen consists of large bundles that are loosely packed and spaced. PSR: Picrosirus Red collagen stain on day 120 (100x). Collagen fiber density increases as fiber color changes from green to yellow to orange. The burn group has an overabundance of immature collagen production (green) while it also has thick and irregularly-arranged yellow to orange fibers in the upper dermis, resembling mature scars. The excision group has primarily yellow to green loose fibers without excessive collagen deposition. $\boldsymbol{\alpha}-\mathrm{SMA}$ (myofibroblasts): myofibroblasts has a delayed increased and persisted well beyond day 90 in the burn group (day 90, 20x). a-SMA (pericytes): Burn group appears to be hypervascular compared to the 
excision group as noted by higher pericytes staining around blood vessels (day 120, 40x).

CD45: The burn group is marked by significantly more leukocyte infiltration starting at day 60 (100x, inset: 400x).

Figure 4. Expression profiles of selected genes in burn and excision groups 7 days after wound creation. Normal distribution was determined with Kolmogorov-Smirnov or D'Agostino-Pearson tests. Data was analyzed with $t$ test and shown as mean with SEM. ${ }^{*} p<0.05,{ }^{* *} p<0.01 . n=6-9$. 
H\&E



엉

MTS

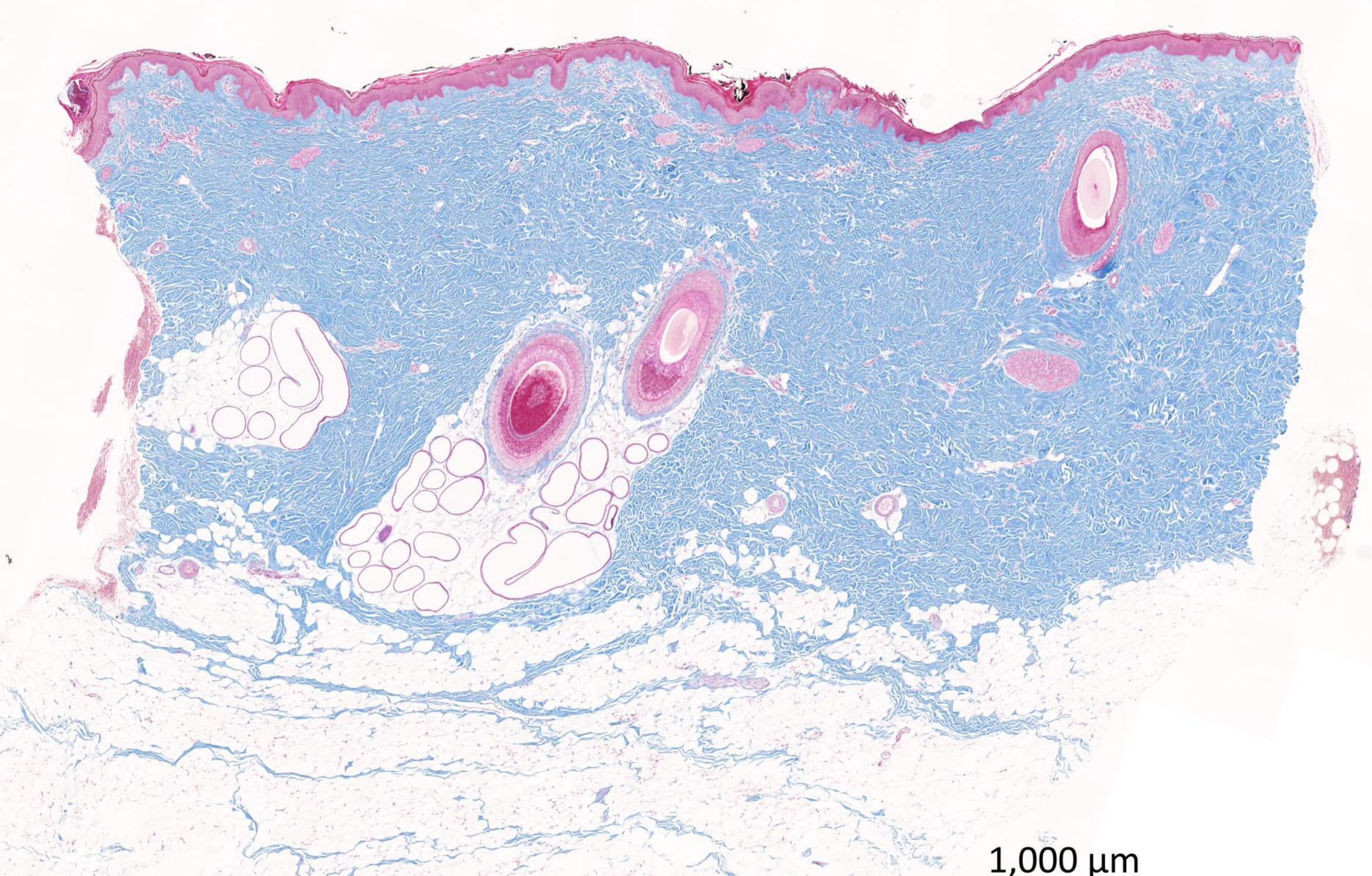

$1,000 \mu \mathrm{mm}$
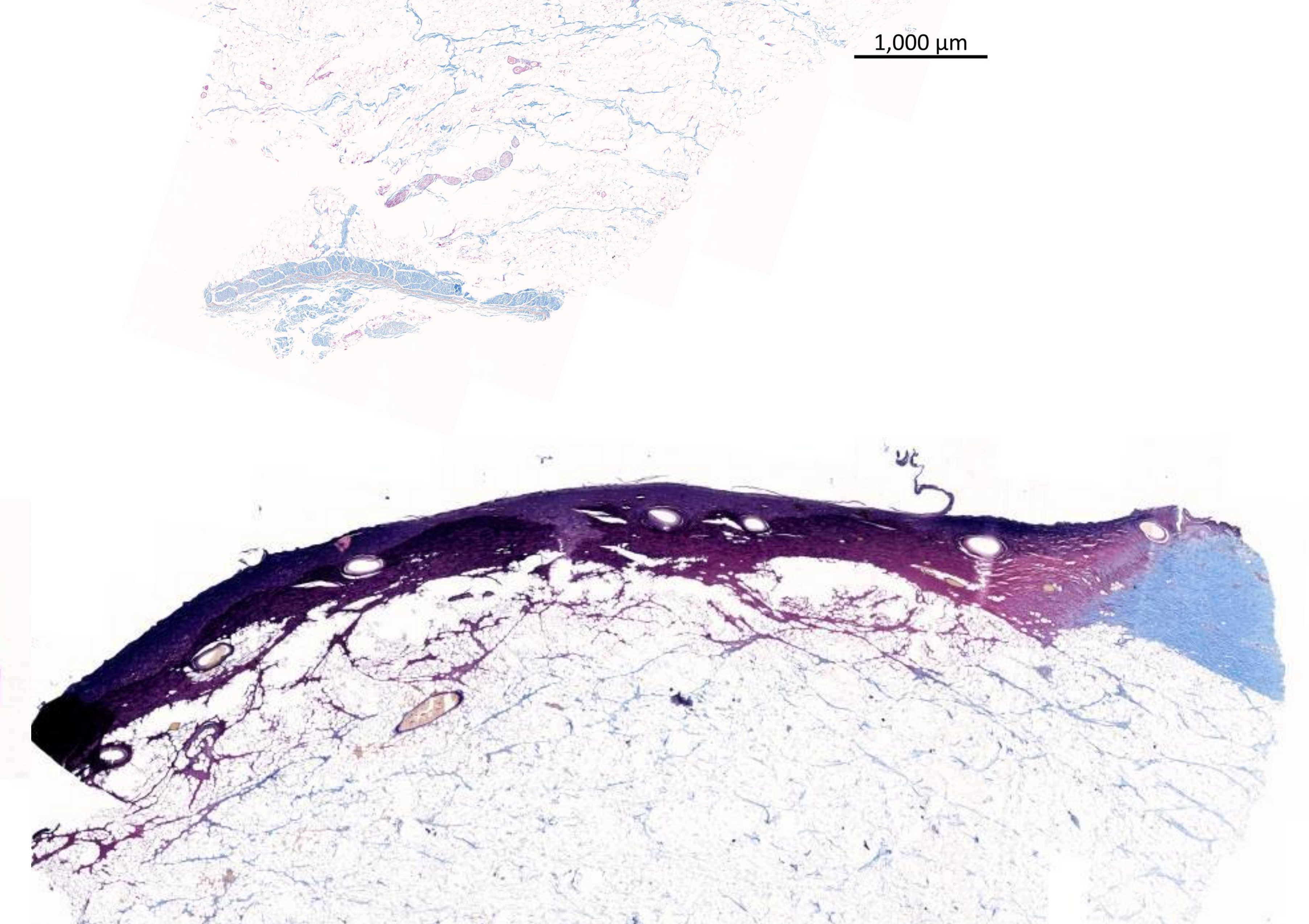

TUNEL

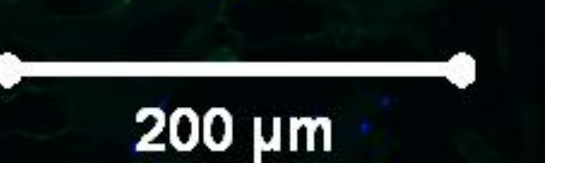

$\underline{1,000 \mu \mathrm{m}}$

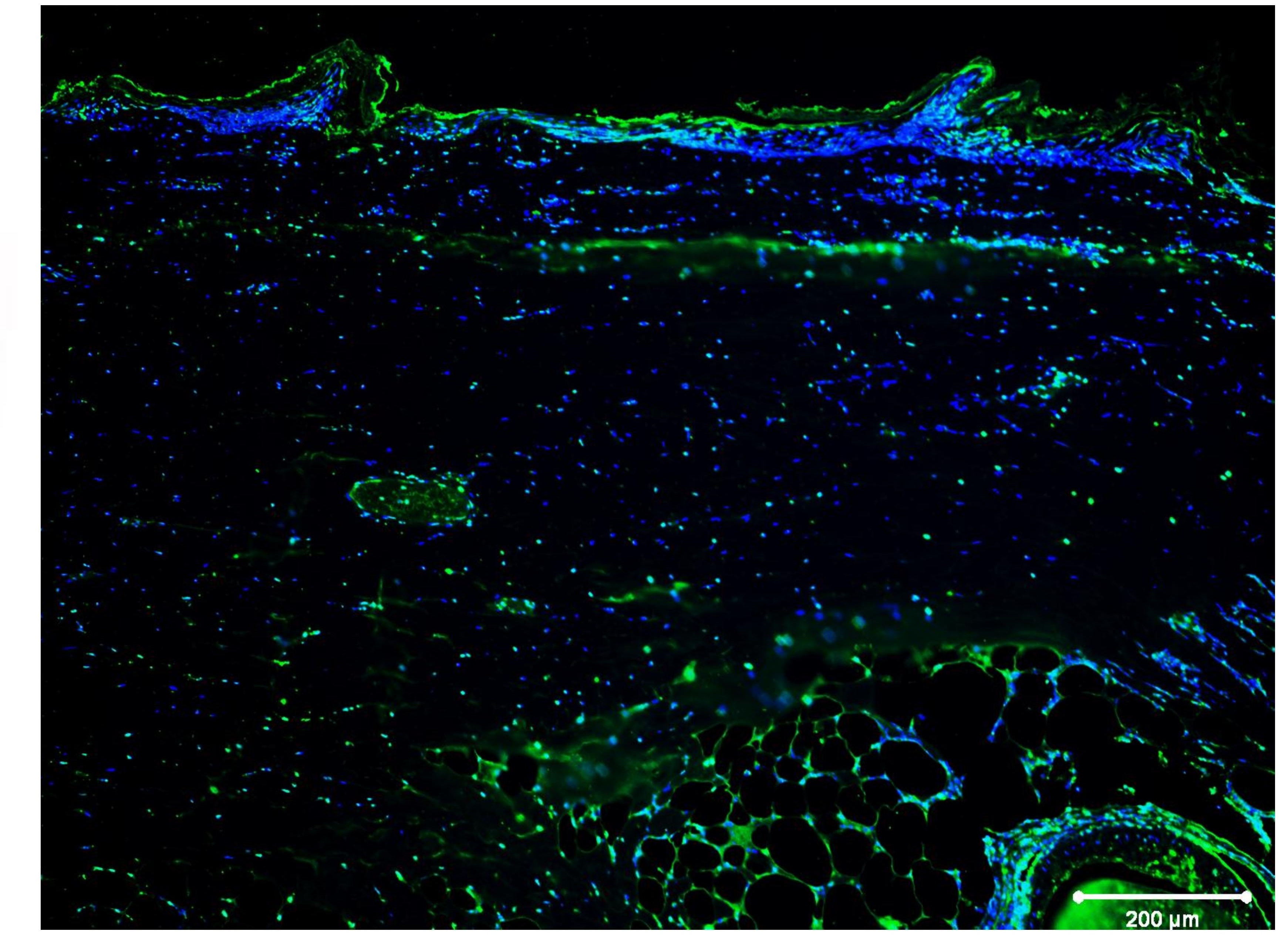




\section{Excised}
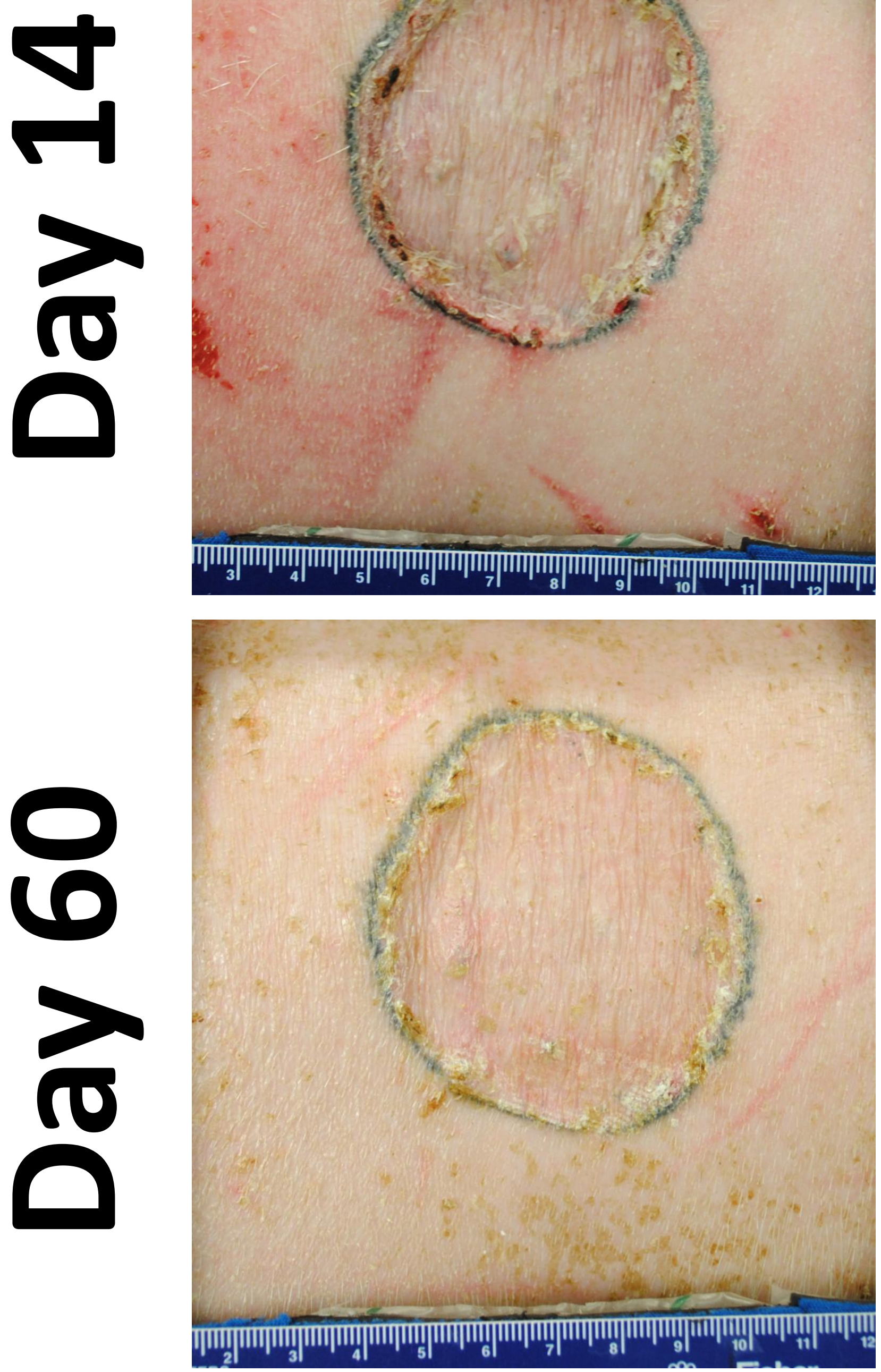

10
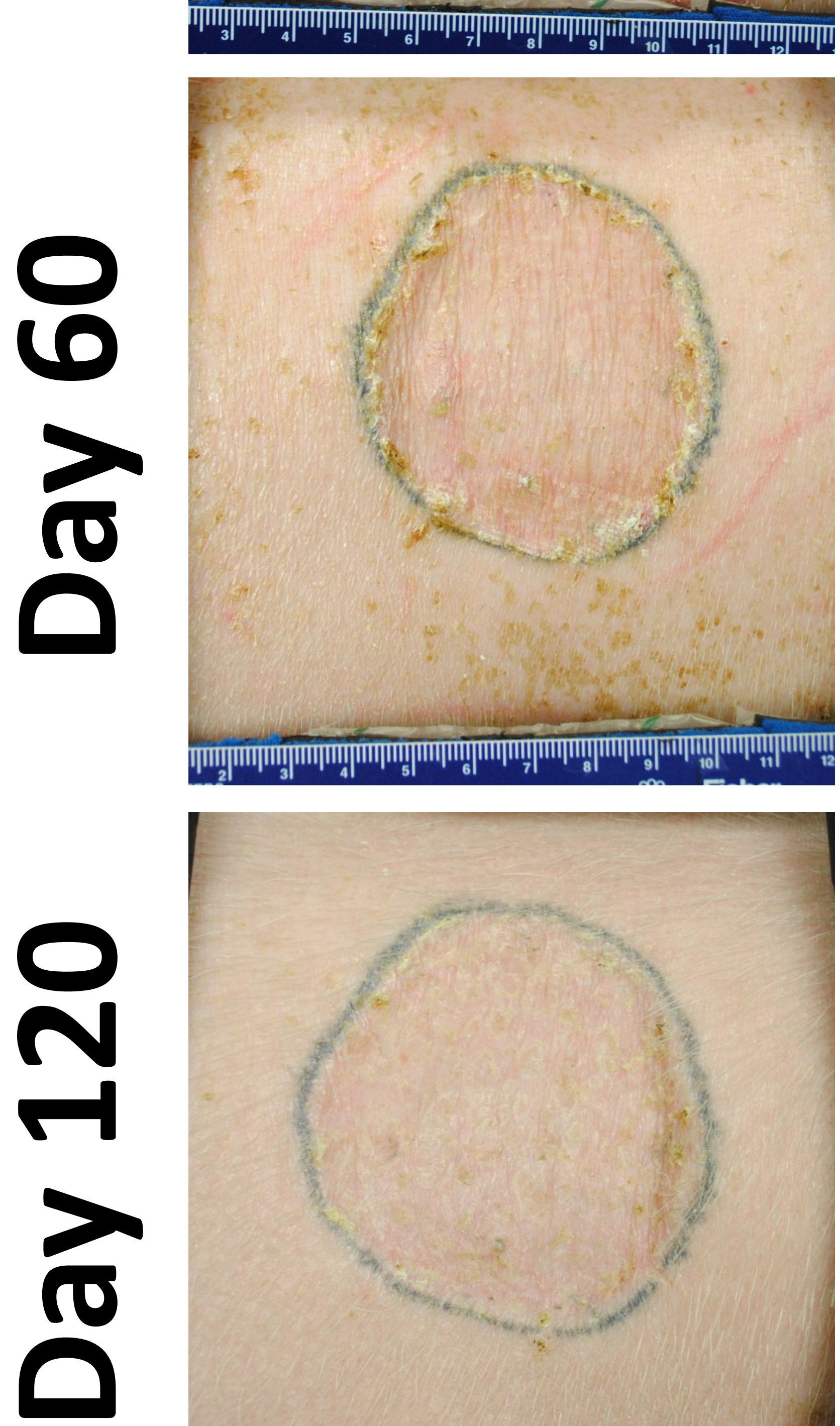

\section{Burned}
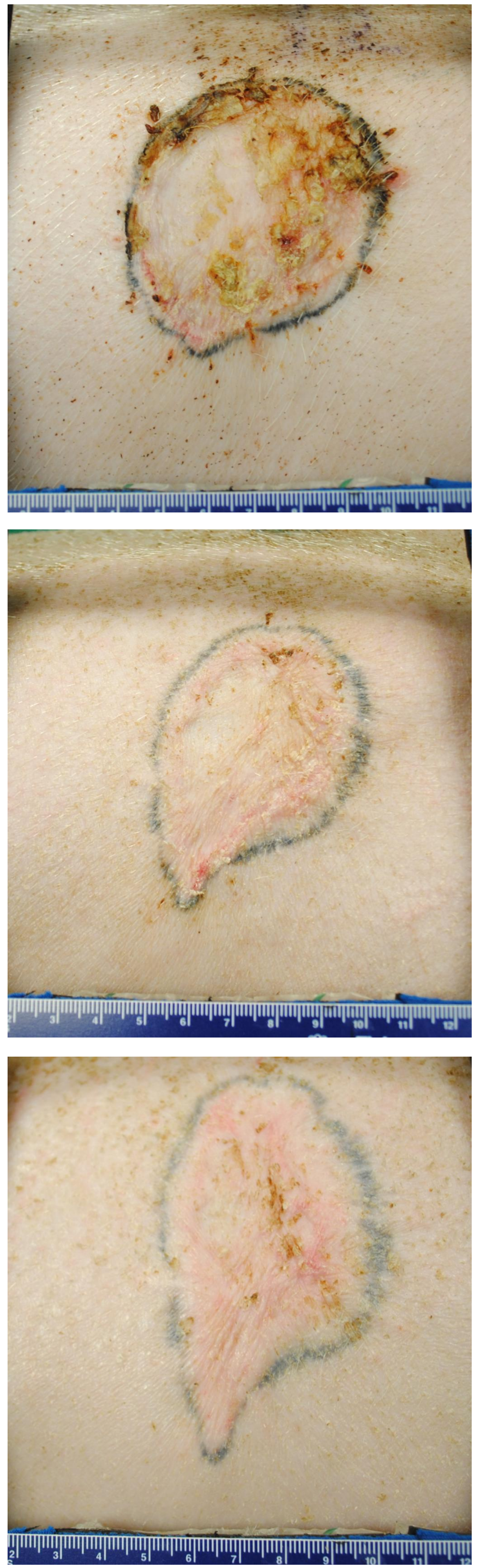


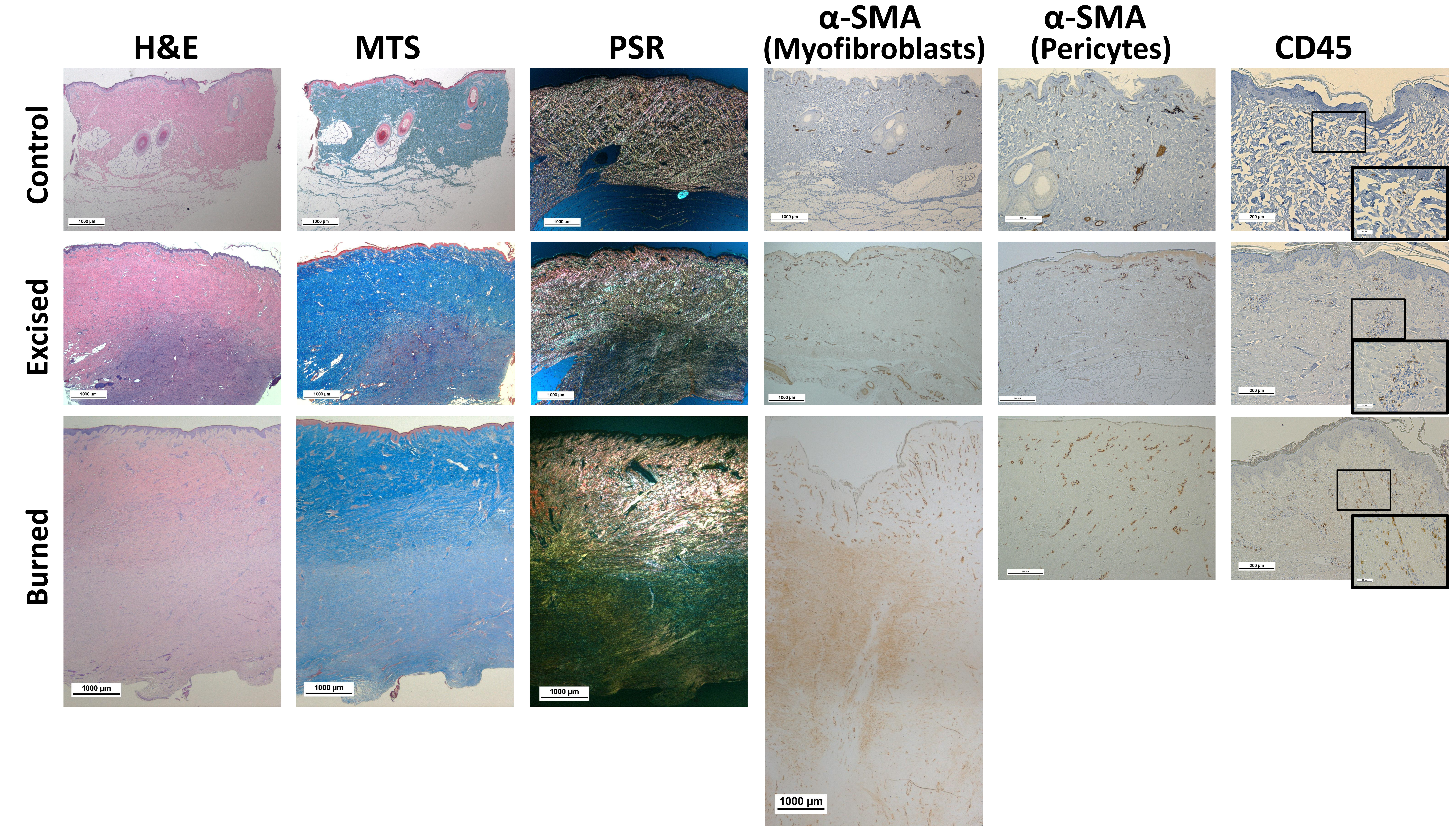




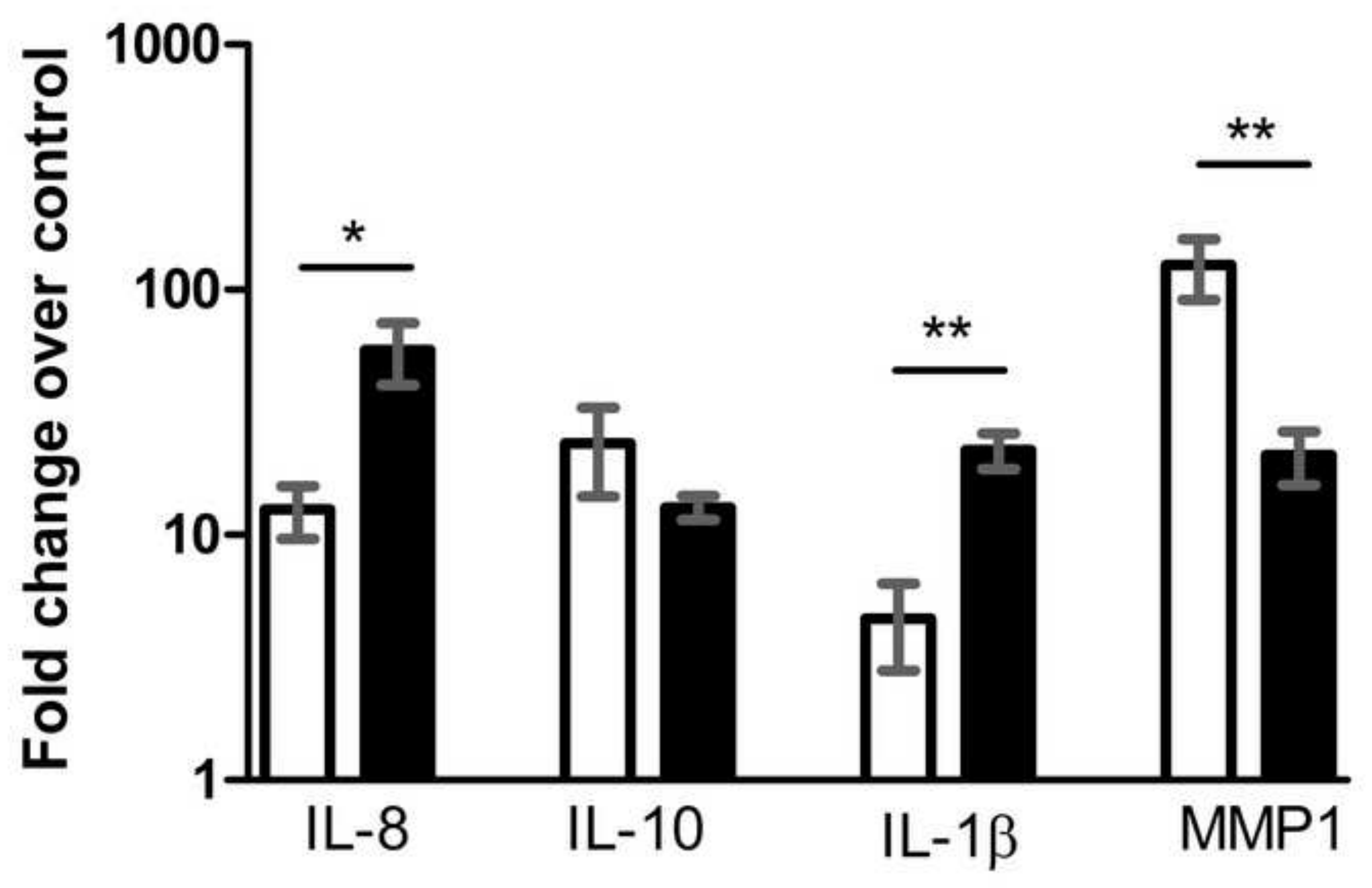

$\square$ Excision $\square$ Burn + Excision 
Table

\begin{tabular}{|c|c|c|c|}
\hline & Excision & Burn & p-value \\
\hline Contracture & $12.03 \% \pm 2.95 \%$ & $28.83 \% \pm 6.18 \%$ & $<0.01$ \\
\hline Distortion & $1.14 \pm 0.05$ & $1.68 \pm 0.13$ & $<0.001$ \\
\hline $\begin{array}{l}\text { Max Graft } \\
\text { Elevation }\end{array}$ & $0.80 \mathrm{~mm} \pm 0.31 \mathrm{~mm}$ & $1.99 \mathrm{~mm} \pm 0.35 \mathrm{~mm}$ & $<0.01$ \\
\hline Scar Index & $0.68 \pm 0.06$ & $0.93 \pm 0.01$ & $<0.01$ \\
\hline Erythema & $7.22 \pm 0.33$ & $10.39 \pm 0.78$ & $<0.01$ \\
\hline Melanin & $36.26 \pm 0.74$ & $41.44 \pm 0.94$ & $<0.01$ \\
\hline POSAS Total & $11.29 \pm 1.01$ & $16.44 \pm 1.43$ & $<0.05$ \\
\hline $\begin{array}{ll}\text { - POSAS } & \text { Vascularity }\end{array}$ & $3.29 \pm 0.41$ & $5.88 \pm 0.62$ & $<0.01$ \\
\hline $\begin{array}{l}\text { - POSAS } \\
\text { Relief }\end{array}$ & $3.92 \pm 0.34$ & $5.93 \pm 0.50$ & $<0.01$ \\
\hline - $\begin{array}{l}\text { POSAS } \\
\text { Mobility }\end{array}$ & $4.08 \pm 0.55$ & $6.20 \pm 0.40$ & $<0.01$ \\
\hline
\end{tabular}

Table 1. The Patient and Observer Scar Assessment Scale (POSAS) were used for assessments of burn and excision groups on day 120 post-grafting. For all parameters higher numbers signify a less desirable outcome. Contraction is reported as the percent change from initial graft size, normalized to growth control. Scar distortion is presented as graft length to width ratio. Scar index is a histological measurement reported as the ratio of scar thickness to normal dermal thickness. Erythema and Melanin are reported as measurements on an arbitrary scale. POSAS are the combined score of individual subjective Vascularity, Relief and Mobility scores described in Materials and Methods. The statistical methods used for the analysis are described under statistical analysis in the result section. Results were considered statistically significant when $\mathrm{p}<0.05 . \mathrm{n}=11-15$. 